\title{
Prenatal drug exposure and maternal and infant feeding behaviour
}

\author{
L L LaGasse, D Messinger, B M Lester, R Seifer, E Z Tronick, C R Bauer, S Shankaran, \\ H S Bada, L L Wright, V L Smeriglio, L P Finnegan, P L Maza, J Liu
}

Arch Dis Child Fetal Neonatal Ed 2003;88:F391-F399

See end of article for authors' affiliations

Correspondence to:

Dr LaGasse, Infant

Development Center,

79 Plain St, Providence,

RI 02903, USA:

linda_lagasse@brown.edu

Accepted

4 November 2002

\begin{abstract}
Objective: To evaluate feeding difficulties and maternal behaviour during a feeding session with 1 month old infants prenatally exposed to cocaine and/or opiates.

Methods: The study is part of the maternal lifestyle study, which recruited 11811 subjects at four urban hospitals, then followed 1388 from 1 to 36 months of age. Exposure to cocaine and opiates was determined by maternal interview and meconium assay. At the 1 month clinic visit, biological mothers were videotaped while bottle feeding their infants. This sample included 364 exposed to cocaine, 45 exposed to opiates, 31 exposed to both drugs, and 588 matched comparison infants. Mothers were mostly black, high school educated, and on public assistance. Videotapes were coded without knowledge of exposure status for frequency, duration and quality of infant sucking, arousal, feeding problems, and maternal feeding activity and interaction.

Results: No cocaine effects were found on infant feeding measures, but cocaine-using mothers were less flexible (6.29 v 6.50), less engaged (5.77 v 6.22), and had shorter feeding sessions (638 v 683 seconds). Opiate exposed infants showed prolonged sucking bursts (29 $v 20$ seconds), fewer pauses (1.6 $v 2.2$ per minute), more feeding problems $(0.55 \vee 0.38)$, and increased arousal (2.59 $v 2.39)$. Their mothers showed increased activity $(30 \vee 22)$, independent of their infants' feeding problems.

Conclusions: Previous concerns about feeding behaviour in cocaine exposed infants may reflect the quality of the feeding interaction rather than infant feeding problems related to prenatal exposure. However, opiate exposed infants and their mothers both contributed to increased arousal and heightened feeding behaviour.
\end{abstract}

F eeding is not only essential for growth, but is a primary context for interactions between infants and their mothers in early infancy. ${ }^{12}$ Early identification of dyads at risk for poor feeding offers an opportunity to intervene in the future health and wellbeing of the infant. ${ }^{1{ }^{34}}$ Evaluation of neonatal feeding has been approached in three ways: clinical reports of dysfunctional sucking, objective (computer assisted) measurement of sucking patterns, and assessments of mother-infant interaction during feeding. Several studies have reported sucking or feeding problems when infants were prenatally exposed to cocaine or opiates, ${ }^{15-12}$ although these observations are controversial. ${ }^{13-15}$ Inconsistent findings may reflect a failure to account for polydrug exposure and sociodemographic factors, ${ }^{5-11}$ small sample sizes with inadequate power to detect subtle effects or low generalisability, ${ }^{1569101215}$ and convenience samples that are not representative of the larger population of drug involved dyads. ${ }^{59}$ Further, no study used methods that could evaluate sucking, symptoms, and mother-infant interaction in the same sample. ${ }^{5-15}$

The purpose of this study was to test the hypotheses that infants exposed to cocaine and/or opiates have abnormal sucking patterns and increased feeding problems and that their mothers are less responsive than non-exposed dyads. One month old infants and their mothers were observed during a feeding session. Three aspects of feeding behaviour were measured: sucking pattern; infant behaviour including problems-for example, spitting up-and crying; and maternal behaviour including feeding related activity-for example, inserting or removing nipple-and interactive behavioursfor example, vocalisation or touches. ${ }^{16}$ The duration of the feeding was also measured, because the end of the session was determined by the mother. The hypotheses were tested with and without adjustment for prenatal exposure to alcohol, tobacco, and marijuana, birth weight, socioeconomic class, and site.

\section{METHODS}

\section{Study design}

The study was part of the Maternal lifestyle study. ${ }^{17}$ The latter was conducted in four major university sites, which are part of the National Institute of Child Health and Human Development Neonatal Research Network (University of Miami, the University of Tennessee at Memphis, Wayne State University and Brown University). The study was conducted in two phases: I, extended through hospital discharge; II, a longitudinal study from 1 month to 36 months (postmenstrual age). The study was approved by the institutional review board at each site.

In phase I, more than 19000 mothers were screened post partum for enrollment between May 1993 and May 1995 at the four sites (see Bauer et $\mathrm{l}^{17}$ for details). About 11800 of the eligible mother-infant dyads agreed to participate. Maternal exclusion criteria included: age < 18 years; identified psychosis or history of institutionalisation for retardation or emotional problems; language barriers that prevented her from giving informed consent or understanding the study. Infant exclusion criteria included: outborn birth; multiple gestation; birth weight < 501 g; gestational age > 42 weeks; the infant was unlikely to survive in the judgment of the attending doctor. A National Institute on Drug Abuse Certificate of Confidentiality was obtained by each site, which ensured confidentiality of information on the subjects' drug use. The certificate superseded the mandatory reporting of illegal substance use that was in effect in the Florida and Rhode Island sites. The certificate was explained to the mother 
during the recruitment, and also informed consent procedure including the condition that the certificate did not exclude reporting of evidence of child abuse or neglect. Drug exposure was determined by self report during a brief interview at recruitment and meconium toxicology with presumptive positive screens confirmed with gas chromatography-mass spectrometry. The assay consisted of an enzyme multiplied immunoassay technique screen for cocaine, opiates, tetrahydrocannabinol, amphetamines, and phencyclidine followed by gas chromatography-mass spectroscopic confirmation of presumptive positive screens (see ElSohly et al $^{18}$ and Lester $e t$ $a l^{19}$ for details). Initial screening for phencyclidine and amphetamine was stopped because of low prevalence.

Phase II was a longitudinal follow up of 1388 exposed and comparison children between 1 month and 3 years of age. It included subjects from phase I with evidence of exposure and matched comparisons who signed a separate consent to participate in the follow up phase of the Maternal lifestyle study. The study definition of "exposure" was the same in phase I and II: maternal admission of cocaine or opiate use during this pregnancy based on the hospital interview or positive gas chromatography-mass spectrometric confirmation of cocaine or opiate metabolites. Opiates were included in the exposed group because of hospital reports indicating that many cocaine users were also using opiates. In phase II, "nonexposed" was defined as denial of cocaine or opiate use during this pregnancy and a negative enzyme multiplied immunoassay technique screen for cocaine and opiate metabolites. A history of maternal alcohol, marijuana, and nicotine use during the pregnancy was recorded during the hospital interview and considered as background variables in both the exposed and non-exposed groups. Use of tranquillisers and barbiturates was less than $2 \%$ and occurred in both groups. Exclusion criteria for phase II were infant chromosomal abnormality or TORCH (toxoplasmosis, other, rubella, cytomegalovirus, herpes virus) infection confirmed before the 1 month visit or if the mother planned to move out of the catchment area. Non-exposed infants were group matched to exposed infants within each centre on race, sex, and gestational age. The 1388 mother/infant dyads (658 in the exposed group and 730 in the comparison group) who came to the 1 month visit were enrolled in the longitudinal study. The uneven groups reflect the replacement of comparison subjects by others if they withdrew consent before the 1 month visit, which was not possible for the fixed population of exposed subjects. The 1 month visit included the feeding procedure.

\section{Subjects in the feeding study}

Subjects were included in this study if they were bottle fed by their biological mother and had a high quality videotape recording $(\mathrm{n}=1028)$. The result was 364 exposed to cocaine, 45 exposed to opiates, 31 exposed to both cocaine and opiates, and 588 comparison infants not exposed to either cocaine or opiates. From the original cohort of 1388, 360 subjects were excluded: 46 were exclusively breast feeding; 70 infants or mothers were unavailable; 118 sessions had technical problems resulting in unscorable videotapes; two infants were more than 46 weeks of postmenstrual age at the time of the feeding session; 100 infants were not cared for by their biological mother; and 24 infants for whom information about their exposure to drugs during pregnancy was unavailable, as mothers failed to complete the interview or refused to answer questions about level of substance use.

\section{Procedure}

Mothers brought their infants to the hospital clinic at 42-46 weeks postmenstrual age (1 month visit) for a neurobehavioural testing battery $y^{20}$ including videotaped bottle feeding. Mothers were instructed to withhold feeding if possible so that the infant would be ready to feed on arrival at the clinic.
However, if the infant was sleeping or recently fed, the feeding procedure was postponed to later in the clinic visit. The feeding session was videotaped through one way mirrors to minimise the intrusiveness of the camera and staff. Mothers were instructed to feed their infants as they would at home and indicate when they were finished. Breast feeding mothers were not asked to participate. The procedure and subsequent scoring were developed and validated with preterm and growth retarded infants, ${ }^{1621}$ and were sufficiently sensitive to detect subtle changes in infant and maternal behaviour after intervention. ${ }^{22}$

Mothers also completed three interviews: the Maternal inventory of substance use (MISU), ${ }^{23}$ which is a detailed interview on the timing and amount of cocaine, opiates, alcohol, nicotine, and marijuana use during pregnancy; socioeconomic status, which is an assessment of the Hollingshead index of social prestige modified for high risk families, ${ }^{24}$ and the Addiction severity index. ${ }^{25}$ The only question from the Addiction severity index used in this study was whether mothers had experienced depression in the preceding 30 days. Infants were weighed and measured by medical staff.

\section{Behavioural scoring}

Table 1 describes the behaviours that were scored from the videotaped feeding session, and the resulting 13 summary scores were used as dependent variables. In accordance with published methods, ${ }^{162}$ research assistants masked to exposure status coded the first 15 minutes of each feeding session for maternal feeding behaviour ("activity" in table 1), maternal interactive behaviour ("stimulation" in table 1), infant sucking (sucking pattern in table 1), feeding problems ("problems" in table 1), and infant crying ("crying" in table 1). All behaviours were frequency counts except for infant sucking, in which case the duration of sucking bursts was coded. A sucking burst was defined as continuous sucking without a pause of more than two seconds. ${ }^{26}$ After observing the entire feeding session regardless of duration, coders completed rating scales that evaluated the level of maternal interaction ("engagement" in table 1), maternal flexibility to her infants' feeding cues ("flexibility" in table 1), infant feeding competence ("competence" in table 1), infant's predominant state or arousal level ${ }^{27}$ ("arousal" in table 1), and the duration of the feeding session ("session" in table 1).

Six coders were trained to reliability on an initial set of 10 videotapes prepared by two "gold standard" coders, coding 10 additional tapes as an initial reliability check. Individual tapes were randomly selected twice a month for continuing reliability checks across coders. Discrepancies between coders were resolved by conferencing and consensus. The $\mathrm{\kappa}$ statistic on the duration of infant sucking was 0.84 , and percentage agreement on the onset and offset of sucking bursts was $68 \%$ and $69 \%$, respectively. Intraclass correlations calculated on the summary scores described below ranged from 0.67 (infant competence) to 0.97 (caretaker activities).

The 13 summary scores in table 1 were based on published methods ${ }^{16}$ with the following exceptions: we measured the duration of sucking bursts, the interburst interval, and duration of the session for greater specificity of the sucking pattern, and we generated two summary scores from the seven maternal scales to distinguish feeding related (flexibility) from interactive (engagement) behaviour. No subjects with short or long feeding sessions were excluded, but frequency data were adjusted for length of the feeding session if less than 15 minutes. Data loss occurred for infant summary scores relevant to sucking pattern and arousal if the infant's mouth or face was obscured $(<5 \%)$. Data loss for engagement occurred when the mother never spoke to the child, as this summary score evaluates the quality rather than the quantity of interaction (2\%). Data loss for interburst interval occurred when there was only a single sucking burst during the feeding 
Table 1 Feeding behaviour summary scores

\begin{tabular}{|c|c|}
\hline Dependent measure & Description \\
\hline \multicolumn{2}{|l|}{ Sucking pattern } \\
\hline Sucking burst & Mean duration (s) of sucking bursts defined as continuous sucking without a pause $>2 s$ \\
\hline Interburst interval & Mean duration (s) between sucking bursts ( $>2 \mathrm{~s}$ pause between sucks) while the nipple was in the infant's mouth \\
\hline Proportion sucking & Proportion of the feeding session during which infant was sucking \\
\hline Bursts per minute & Number of sucking bursts per minute \\
\hline \multicolumn{2}{|l|}{ Infant behaviour } \\
\hline Problems & $\begin{array}{l}\text { Frequency of feeding problems. Summed proportion (adjusted for length of feed and multiplied by } 100 \text { ) of refusal and rejection of } \\
\text { the nipple, dribbling milk, hiccoughs, spitting up, and coughs }\end{array}$ \\
\hline Crying & Frequency of crying (continuous cries were coded every $2 \mathrm{~s}$ ). Proportion of the session (multiplied by 100 ) when infant was crying \\
\hline Competence & $\begin{array}{l}\text { Average of three nine-point scales: appropriateness of state for feeding, ease of feeding, and infant's responsiveness }(\alpha=0.62) \text {. } \\
\text { High scores indicate greater competence }\end{array}$ \\
\hline Arousal & $\begin{array}{l}\text { Predominant state or level of arousal of the infant rating scale along a four-point scale: sleeping, drowsy, alert, fussy/crying (state } \\
\text { definitions of the Brazelton scale) }\end{array}$ \\
\hline \multicolumn{2}{|r|}{ 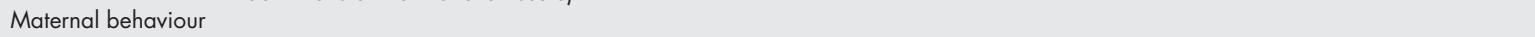 } \\
\hline Activity & $\begin{array}{l}\text { Frequency of maternal feeding activity. Summed proportion (adjusted for length of feed and multiplied by } 100 \text { ) of stimulates } \\
\text { rooting, inserts nipple, stimulates sucking with the bottle, removes nipple, burps infant, wipes mouth, iiggles the infant, repositions } \\
\text { the infant, and checks amount taken }\end{array}$ \\
\hline Stimulation & $\begin{array}{l}\text { Frequency of maternal stimulation or interactive behaviour. Summed proportion (adjusted for length of feed and multiplied by 100) } \\
\text { of vocalisations, and stroking/caressing and kissing the infant }\end{array}$ \\
\hline Engagement & $\begin{array}{l}\text { Rating of level of engagement with her infant. Average of four nine-point scales including quality of attentiveness, quality of } \\
\text { physical contact, delight in baby, and quality of vocalisation }(\alpha=0.79) \text {. High scores indicate greater engagement }\end{array}$ \\
\hline Flexibility & $\begin{array}{l}\text { Rating of maternal flexibility to infants' feeding cues. Average of three nine-point scales including determination of amount, degree } \\
\text { of confidence, and appropriateness of behaviour }(\alpha=0.69) \text {. High scores indicate greater flexibility/low scores indicate more } \\
\text { maternal control of the feeding session }\end{array}$ \\
\hline Session & Duration (s) of coded feeding session up to 15 minutes \\
\hline
\end{tabular}

session $(<4 \%)$. There was no association between prenatal exposure to cocaine or opiates and data loss in these summary scores.

\section{Statistical analysis}

$t$ Tests and $\chi^{2}$ statistics were used to compare the cocaine and/or opiate exposed and comparison groups on medical and other characteristics. The 13 dependent measures in table 1 were tested with four sets of analyses. Analysis 1 was a two way analysis of variance testing two factors: any cocaine exposure (exposed/non-exposed) and any opiate exposure (exposed/non-exposed). The analysis of variance (type III sum of squares) tests each factor after adjustment for the other. The cocaine by opiate interaction was included in the model only if it was significant. This approach yielded tests of exposure effects in the primary analysis, avoiding post hoc analysis on the primary study questions. Analysis 2 was a two way analysis of variance testing cocaine and opiate effects after adjustment for covariates described below. Analysis 3 was a univariate analysis of heavy cocaine exposure, some cocaine exposure, and no exposure. Heavy cocaine exposure was defined as at least three days a week during the first trimester. ${ }^{28}$ Any other cocaine exposure was considered some exposure. These analyses were conducted on a subset of subjects (873 of 1028) who admitted cocaine use on the MISU and who did not use opiates during pregnancy based on initial hospital interview, toxicology, or self report on the MISU. Opiate users were excluded because opiate use could co-occur with heavy, some, or no cocaine use and potentially confound level of cocaine exposure effects. Analysis 4 was a one way analysis of variance testing the three levels of cocaine exposure groups after adjustment for covariates described below. Student's Newman-Keuls tests were used for post hoc analysis of significant effects for level of cocaine exposure.

\section{Covariates}

In analyses 2 and 4, covariates were included either because of relevance to drug-using populations-for example, socioeconomic status, birth weight, polydrug use-or because they met the following statistical criteria: the variable correlated with both drug exposure and feeding behaviour $(\mathrm{p}<0.05)$, and did not correlate highly with other covariates (Pearson $r<0.70) .{ }^{29-32}$ The variables in tables 2 and 3 were examined for possible inclusion as covariates because feeding behaviour has been associated with maternal age, ${ }^{23}$ depression, ${ }^{34}{ }^{35}$ low income, ${ }^{2}$ socioeconomic status, ${ }^{33}$ maternal education, ${ }^{23}$ parity (primiparous versus multiparous), ${ }^{23}$ race $^{12}{ }^{2}$ infant's age, ${ }^{36}$ and birth weight. ${ }^{23}$ The maternal and infant characteristics that met the criteria and were used in the adjusted analyses included: socioeconomic status, ${ }^{24}$ birth weight, conceptional age (gestational + chronological age), maternal age, race, site, and other drug use.

\section{Covariates for other drug use}

The pattern of drug use reported by the mother on the MISU was used to generate covariates for alcohol, marijuana, and tobacco by averaging use per day over the three trimesters of pregnancy. The average for each drug use variable was reduced to three categories of exposure (heavy, some, and none). Cut offs for heavy exposure were based on published thresholds for medical as well as neurobehavioural outcome. ${ }^{28}{ }^{37}$ For alcohol, heavy exposure was $\geqslant 0.5 \mathrm{oz}$ of absolute alcohol a day (one standard drink). For marijuana, heavy exposure was defined as $\geqslant 0.5$ joints a day. For tobacco, heavy exposure was defined as $\geqslant 10$ cigarettes a day. Each three category drug variable was used to construct two covariates that tested threshold effects: (a) the contrast between heavy and some/no exposure; $(b)$ the contrast between some and no exposure. For example, if the contrast between some and no exposure was significant but not the contrast between heavy and some/no exposure, the interpretation would be that the threshold for the effect is at the cut off for some exposure and there is no additional effect for heavy exposure. This approach eliminated the need for post hoc analysis. A separate covariate was included for any occurrence of binge drinking (more than five drinks at one time during pregnancy). Thus, other drug use was measured by seven covariates (two for each drug and the binge measure).

\section{RESULTS}

Characteristics of the exposed and non-exposed infants and their mothers

Table 2 gives the characteristics of infants exposed to cocaine and/or opiates and comparison infants. Cocaine exposed 
Table 2 Characteristics of cocaine/opiate exposed and comparison groups

\begin{tabular}{|c|c|c|c|c|c|c|}
\hline & \multicolumn{3}{|l|}{ Cocaine } & \multicolumn{3}{|l|}{ Opiate } \\
\hline & $\begin{array}{l}\text { Exposed } \\
(n=395)\end{array}$ & $\begin{array}{l}\text { Non-exposed } \\
(n=633)\end{array}$ & p Value & $\begin{array}{l}\text { Exposed } \\
(n=76)\end{array}$ & $\begin{array}{l}\text { Non-exposed } \\
(n=952)\end{array}$ & p Value \\
\hline Chronological age (weeks) & $6.59(3.26)$ & 7.18 (3.49) & 0.008 & $6.76(3.62)$ & $6.97(3.40)$ & 0.609 \\
\hline Conceptional age (weeks) & $42.98(2.33)$ & 43.54 (2.09) & 0.001 & $43.51(2.13)$ & $43.31(2.20)$ & 0.442 \\
\hline Best $O B$ gestational age (weeks) & $36.39(3.80)$ & $36.36(3.95)$ & 0.900 & 36.75 (3.85) & 36.34 (3.90) & 0.378 \\
\hline Birth weight (g) & $2588(721)$ & $2672(841)$ & 0.098 & 2658 (823) & $2638(796)$ & 0.831 \\
\hline Length $(\mathrm{cm})$ & $46.62(4.60)$ & $46.96(5.07)$ & 0.277 & $46.76(5.16)$ & $46.83(4.87)$ & 0.910 \\
\hline Head circumference $(\mathrm{cm})$ & $32.10(2.67)$ & $32.24(3.04)$ & 0.469 & $32.16(2.98)$ & $32.19(2.90)$ & 0.950 \\
\hline Apgar at $1 \mathrm{~min}$ (median) & 8 & 8 & & 8 & 8 & \\
\hline Apgar at $5 \mathrm{~min}$ (median) & 9 & 9 & & 9 & 9 & \\
\hline Male (\%) & 51.6 & 51.7 & 0.997 & 56.6 & 51.3 & 0.372 \\
\hline
\end{tabular}

Unless otherwise indicated, values are mean (SD). $p$ Values in bold are significant.

$\mathrm{OB}$, Obstetrical estimate.

infants were about 4 days younger at the time of assessment by both chronological and conceptional age $(p=0.008$ and $\mathrm{p}=0.001$ respectively). There were no significant differences between the groups for gestational age (range 22-42 weeks), weight (range 520-4440 g), length, and head circumference at birth, Apgar scores, and sex. All infants were feeding in room air during the feeding session.

Table 3 gives maternal characteristics. Most study participants were black, unmarried, Medicaid recipients with two thirds of the sample falling below the federal poverty line, and high school educated. Mothers who used cocaine were more likely to be low socioeconomic status $(p=0.001)$, older $(\mathrm{p}=0.001)$, unmarried $(\mathrm{p}=0.001), \mathrm{a}$ Medicaid recipient $(p=0.001)$, below the poverty line $(p=0.001)$, less educated $(\mathrm{p}<0.001)$, without prenatal care $(\mathrm{p}<0.001)$, depressed $(\mathrm{p}=0.038)$, and multiparous $(\mathrm{p}=0.001)$ than mothers in the comparison group. Opiate-using mothers were more likely to be older $(p=0.001)$, white $(p=0.001)$, and above the poverty line $(p=0.001)$ than the comparison group.

\section{Cocaine exposure}

Table 4 shows the means for the study groups and results of analysis 1 (unadjusted) and analysis 2 (adjusted for covariates). The adjusted marginal means from analysis 2 are presented for the main effects of cocaine exposure and opiate exposure. There were no significant differences between cocaine exposed and non-exposed infants in measures of sucking pattern (sucking burst, interburst interval, proportion of sucking, and bursts per minute), feeding competence, feeding problems, crying, and level of arousal. However, mothers of cocaine exposed infants showed less engagement (unadjusted, $\mathrm{p}=0$.003; adjusted, $\mathrm{p}=0.004)$, and less flexibility (unadjusted, $\mathrm{p}=0.022$; adjusted, $\mathrm{p}=0.024$ ), and had a shorter feeding session (unadjusted, $\mathrm{p}=0$.001; adjusted, $\mathrm{p}=0.010$ ) than mothers of non-exposed infants.

\section{Opiate exposure}

Opiate exposed infants showed longer sucking bursts (unadjusted, $\mathrm{p}=0.044$; adjusted, $\mathrm{p}=0.004)$, fewer bursts per minute (unadjusted, $\mathrm{p}=0.001$; adjusted $\mathrm{p}=0.001$ ), more feeding problems (unadjusted, $\mathrm{p}=0.031$; adjusted $\mathrm{p}=0.028$ ), and higher arousal (unadjusted, $\mathrm{p}=0.002$; adjusted, $\mathrm{p}=0.021$ ) than non-exposed infants. Mothers of opiate exposed infants showed more feeding activity (unadjusted, $\mathrm{p}=0.001$; adjusted, $\mathrm{p}=0.001$ ) than mothers of nonexposed infants. Increased stimulation (unadjusted, $\mathrm{p}=0.003$ ) and less maternal flexibility (unadjusted, $\mathrm{p}=0.017$ ) were found in the analysis without covariates, but these effects were not significant when adjusted for covariates ( $p>0.05$ in both cases).

The findings of longer and fewer sucking bursts, increased problems and arousal in opiate exposed infants, and more activity by their mothers raise the possibility that maternal behaviour may account for infant behaviour rather than opiate exposure per se. To test this hypothesis, infant measures that showed significant opiate effects were reanalysed with adjustment for maternal activity in the context of the other covariates. The main effects for opiate exposure on the duration of sucking bursts $(\mathrm{p}=0.018)$ and bursts per minute $(\mathrm{p}=0.042)$ were maintained, but the opiate effects on

Table 3 Maternal characteristics of cocaine/opiate exposed and comparison groups

\begin{tabular}{|c|c|c|c|c|c|c|}
\hline & \multicolumn{3}{|l|}{ Cocaine } & \multicolumn{3}{|l|}{ Opiate } \\
\hline & $\begin{array}{l}\text { Exposed } \\
(n=395)\end{array}$ & $\begin{array}{l}\text { Non-exposed } \\
(n=633)\end{array}$ & $\mathrm{p}$ Value & $\begin{array}{l}\text { Exposed } \\
(n=76)\end{array}$ & $\begin{array}{l}\text { Non-exposed } \\
(n=952)\end{array}$ & $\mathrm{p}$ Value \\
\hline SES & $25.90(9.44)$ & $28.31(9.81)$ & 0.001 & $27.50(9.08)$ & $27.37(9.79)$ & 0.910 \\
\hline Maternal age & $30.47(4.87)$ & $26.39(5.80)$ & 0.001 & $30.62(6.68)$ & $27.74(5.68)$ & 0.001 \\
\hline Race & & & & & & 0.001 \\
\hline$\%$ Black & 83.0 & 78.8 & 0.352 & 60.5 & 82.0 & \\
\hline$\%$ White & 10.6 & 13.9 & & 30.3 & 11.2 & \\
\hline$\%$ Hispanic & 5.3 & 6.5 & & 6.6 & 6.0 & \\
\hline$\%$ Other (non-Hispanic) & 1.0 & 0.8 & & 2.6 & 0.7 & \\
\hline$\%$ Single & 90.1 & 78.2 & 0.001 & 78.9 & 83.1 & 0.360 \\
\hline$\%$ No private insurance ${ }^{*}$ & 91.1 & 81.4 & 0.001 & 82.9 & 85.3 & 0.572 \\
\hline$\%$ Below federal poverty line & 78.6 & 63.2 & 0.001 & 70.9 & 45.7 & 0.001 \\
\hline$\%<$ high school degree/GED & 48.4 & 32.1 & 0.001 & 32.9 & 38.8 & 0.308 \\
\hline$\%$ No prenatal care & 19.7 & 4.3 & 0.001 & 9.2 & 10.3 & 0.764 \\
\hline$\%$ Depression in past 30 days & 24.0 & 18.5 & 0.038 & 23.0 & 20.4 & 0.602 \\
\hline$\%$ Multiparous & 91.6 & 69.5 & 0.001 & 75.0 & 78.3 & 0.509 \\
\hline
\end{tabular}

Unless otherwise indicated, values are mean (SD). $p$ Values in bold are significant.

* Insurance status unknown for eight exposed and six comparison subjects.

SES, Socioeconomic status; GED, general educational development. 
Table 4 Feeding measures in cocaine and opiate exposed and non-exposed infants

\begin{tabular}{|c|c|c|c|c|c|c|c|c|c|c|c|c|}
\hline \multirow[b]{3}{*}{ Measure } & \multicolumn{6}{|c|}{ Cocaine } & \multicolumn{6}{|c|}{ Opiate } \\
\hline & \multicolumn{2}{|c|}{ Exposed } & \multicolumn{2}{|c|}{ Non-exposed } & \multicolumn{2}{|l|}{$\mathrm{p}$ Value } & \multicolumn{2}{|c|}{ Exposed } & \multicolumn{2}{|c|}{ Non-exposed } & \multicolumn{2}{|l|}{$P=$} \\
\hline & $N$ & Mean (SE) & $N$ & Mean (SE) & Unadj. & Adj. & $N$ & Mean (SE) & $N$ & Mean (SE) & Unadj. & Adj. \\
\hline \multicolumn{13}{|l|}{ Sucking pattern } \\
\hline Sucking burst & 378 & $24.19(2.22)$ & 603 & $24.73(1.92)$ & 0.816 & 0.870 & 74 & $29.27(3.25)$ & 907 & $19.64(1.04)$ & 0.044 & 0.004 \\
\hline Interburst interval & 364 & $6.64(0.50)$ & 582 & $5.88(0.40)$ & 0.714 & 0.233 & 68 & $6.38(0.60)$ & 878 & $6.15(0.18)$ & 0.990 & 0.718 \\
\hline Proportion sucking & 378 & $0.46(0.01)$ & 603 & $0.45(0.01)$ & 0.932 & 0.427 & 74 & $0.45(0.02)$ & 907 & $0.46(0.01)$ & 0.266 & 0.734 \\
\hline Bursts per minute & 378 & $1.94(0.10)$ & 603 & $1.88(0.09)$ & 0.997 & 0.594 & 74 & $1.62(0.15)$ & 907 & $2.19(0.05)$ & 0.001 & 0.001 \\
\hline \multicolumn{13}{|l|}{ Infant behaviour } \\
\hline Competence & 386 & $5.08(0.11)$ & 620 & $5.19(0.10)$ & 0.989 & 0.318 & 74 & $5.16(0.17)$ & 932 & $5.11(0.05)$ & 0.979 & 0.801 \\
\hline Problems & 395 & $0.44(0.05)$ & 633 & $0.49(0.05)$ & 0.602 & 0.294 & 76 & $0.55(0.08)$ & 952 & $0.38(0.02)$ & 0.031 & 0.028 \\
\hline Crying & 395 & $1.03(0.20)$ & 633 & $0.68(0.17)$ & 0.958 & 0.080 & 76 & $0.86(0.29)$ & 952 & $0.85(0.09)$ & 0.493 & 0.962 \\
\hline Arousal* & 389 & $2.57(0.07)$ & 621 & $2.41(0.06)$ & 0.154 & 0.091 & 75 & $2.59(0.09)$ & 935 & $2.39(0.03)$ & 0.002 & 0.021 \\
\hline \multicolumn{13}{|l|}{ Maternal behaviour } \\
\hline Activity & 395 & $25.71(1.29)$ & 633 & $26.61(1.13)$ & 0.588 & 0.490 & 76 & 30.48 (1.91) & 952 & $21.83(0.61)$ & 0.001 & 0.001 \\
\hline Stimulation* & 395 & $5.05(0.61)$ & 633 & $6.30(0.49)$ & 0.258 & 0.109 & 76 & $6.11(0.74)$ & 952 & $5.25(0.23)$ & 0.003 & 0.252 \\
\hline Engagement* & 388 & $5.77(0.12)$ & 619 & $6.22(0.10)$ & 0.003 & 0.004 & 76 & $5.98(0.15)$ & 931 & $6.02(0.05)$ & 0.932 & 0.821 \\
\hline Flexibility* & 395 & $6.29(0.09)$ & 633 & $6.50(0.08)$ & 0.002 & 0.024 & 76 & $6.29(0.14)$ & 952 & $6.50(0.04)$ & 0.017 & 0.128 \\
\hline Session & 395 & 637.75 (17.28) & 633 & $682.96(15.11)$ & 0.001 & 0.010 & 76 & $650.72(25.48)$ & 952 & $669.99(8.11)$ & 0.714 & 0.460 \\
\hline
\end{tabular}

arousal $(\mathrm{p}=0.132)$ and feeding problems $(\mathrm{p}=0.051)$ failed to reach significance. These findings suggest that the behaviour of opiate exposed infants was at least in part independent of maternal behaviour.

\section{Interaction of cocaine and opiate exposure}

There were significant interaction effects of cocaine and opiate exposure on arousal (unadjusted, $\mathrm{p}=0.017$; adjusted, $\mathrm{p}=0.018$ ), stimulation (unadjusted, $\mathrm{p}=0.035$; adjusted, $\mathrm{p}=0.029$ ), engagement (unadjusted, $\mathrm{p}=0.014$; adjusted, $\mathrm{p}=0.039$ ), and flexibility (unadjusted, $\mathrm{p}=0.037$, only). Tables 5 (unadjusted) and 6 (adjusted) show the main effect differences for cocaine exposure and opiate exposure by exposure to the other drug for these measures.

Cocaine exposed infants also exposed to opiates showed higher arousal than non-exposed infants (unadjusted, $\mathrm{p}=0.047$; adjusted $\mathrm{p}=0.032)$. Similarly, opiate exposed infants also exposed to cocaine showed higher arousal than non-exposed infants. No differences in arousal were shown for single drug exposure.

Mothers of cocaine exposed infants who used opiates showed less stimulation than mothers of non-exposed infants, but only in the adjusted analysis (unadjusted, $p=0.092$; adjusted, $p=0.045$ ). Cocaine-using mothers who did not use opiates showed no differences in stimulation. Mothers of opiate exposed infants who did not use cocaine showed more stimulation than mothers of non-exposed infants (unadjusted, $\mathrm{p}=0.001$; adjusted, $\mathrm{p}=0.01$ ). But opiate-using mothers who used cocaine showed no differences in stimulation.

Cocaine-using mothers who used opiates showed less engagement than mothers who did not use these drugs (unadjusted, $\mathrm{p}=0.005$; adjusted, $\mathrm{p}=0.008$ ), whereas mothers only using cocaine showed no differences (unadjusted, $\mathrm{p}=0.343$; adjusted, $\mathrm{p}=0.131$ ). There were no effects of opiate use on engagement with or without cocaine use.

Cocaine-using mothers who used opiates showed less flexibility than mothers who did not use either drug (unadjusted, $p=0.008$ ). Similarly, opiate-using mothers who also used cocaine showed less flexibility. No differences in flexibility were shown for single drug use. The interaction was not significant in the adjusted analysis of flexibility $(\mathrm{p}=0.069)$.

Table 5 Unadjusted main effect differences for interactions of cocaine and opiate exposure

\begin{tabular}{|c|c|c|c|c|c|c|c|c|}
\hline \multirow[b]{2}{*}{ Measure } & \multicolumn{4}{|l|}{ Cocaine exposed } & \multicolumn{4}{|l|}{ Opiate exposed } \\
\hline & Opiate exposed & $\mathrm{p}$ Value & Opiate non-exposed & $\mathrm{p}$ Value & Cocaine exposed & $\mathrm{p}$ Value & Cocaine non-exposed $p$ & p Value \\
\hline Arousal & $0.33(0.17)$ & 0.047 & $-0.08(0.05)$ & 0.081 & $0.47(0.13)$ & 0.001 & $0.06(0.11)$ & 0.612 \\
\hline Stimulation & $-2.48(1.47)$ & 0.092 & $0.75(0.42)$ & 0.077 & $0.64(1.18)$ & 0.589 & $3.87(0.98)$ & 0.001 \\
\hline Engagement & $-0.80(0.28)$ & 0.005 & $-0.08(0.08)$ & 0.343 & $-0.37(0.23)$ & 0.100 & $0.35(0.09)$ & 0.064 \\
\hline Flexibility & $-0.71(0.27)$ & 0.008 & $-0.13(0.08)$ & 0.085 & $-0.62(0.21)$ & 0.004 & $-0.04(0.18)$ & 0.810 \\
\hline
\end{tabular}

Values are mean difference (SE). pValues in bold are significant.

Table 6 Adjusted main effect differences for interactions of cocaine and opiate exposure

\begin{tabular}{|c|c|c|c|c|c|c|c|c|}
\hline \multirow[b]{2}{*}{ Measure } & \multicolumn{4}{|l|}{ Cocaine exposed } & \multicolumn{4}{|l|}{ Opiate exposed } \\
\hline & Opiate exposed & $\mathrm{p}$ Value & Opiate non-exposed & $\mathrm{p}$ Value & Cocaine exposed & $\mathrm{p}$ Value & Cocaine non-exposed & $\mathrm{p}$ Value \\
\hline Arousal & $0.36(0.17)$ & 0.032 & $-0.05(0.06)$ & 0.431 & $0.41(0.13)$ & 0.002 & $0.01(0.11)$ & 0.987 \\
\hline Stimulation & $-2.85(1.42)$ & 0.045 & $0.35(0.51)$ & 0.494 & $-0.74(1.14)$ & 0.517 & $2.47(0.95)$ & 0.010 \\
\hline Engagement & $-0.75(0.28)$ & 0.008 & $-0.15(0.10)$ & 0.131 & $-0.33(0.23)$ & 0.141 & $0.26(0.19)$ & 0.161 \\
\hline
\end{tabular}

Values are mean difference (SE). pValues in bold are significant. Note: The interaction of cocaine and opiate exposure on flexibility was not significant after adjustment for covariates. 
Table 7 Feeding measures in groups with heavy cocaine exposure, some cocaine exposure, and no exposure

\begin{tabular}{|c|c|c|c|c|c|c|c|c|}
\hline \multirow[b]{2}{*}{ Measure } & \multicolumn{2}{|c|}{ Heavy } & \multicolumn{2}{|c|}{ Some } & \multicolumn{2}{|c|}{ None } & \multicolumn{2}{|l|}{$\mathrm{p}$ Value } \\
\hline & $\mathrm{N}$ & Mean (SE) & $\mathrm{N}$ & Mean (SE) & $\mathrm{N}$ & Mean (SE) & Unadj. & Adj. \\
\hline \multicolumn{9}{|l|}{ Sucking pattern } \\
\hline Sucking burst & 96 & $22.10(2.92)$ & 182 & $18.48(2.21)$ & 555 & 20.61 (1.37) & 0.510 & 0.505 \\
\hline Interburst interval & 91 & $5.40(0.54)$ & 177 & $6.03(0.41)$ & 538 & $6.43(0.25)$ & 0.450 & 0.253 \\
\hline Proportion sucking & 96 & $0.48(0.02)$ & 182 & $0.47(0.01)$ & 555 & $0.45(0.01)$ & 0.918 & 0.370 \\
\hline $\begin{array}{l}\text { Bursts per minute } \\
\text { Infant behaviour }\end{array}$ & 96 & $2.25(0.14)$ & 182 & $2.36(0.11)$ & 555 & $2.12(0.07)$ & 0.649 & 0.212 \\
\hline Competence & 94 & $5.09(0.16)$ & 188 & $4.87(0.12)$ & 573 & $5.15(0.07)$ & 0.172 & 0.128 \\
\hline Problems & 97 & 0.39 (0.08) & 191 & $0.38(0.06)$ & 585 & $0.39(0.04)$ & 0.981 & 0.975 \\
\hline Crying & 97 & $0.97(0.25)$ & 191 & $0.79(0.19)$ & 585 & $0.76(0.12)$ & 0.404 & 0.749 \\
\hline Arousal & 95 & $2.31(0.08)$ & 189 & $2.34(0.06)$ & 574 & $2.41(0.04)$ & 0.040 & 0.468 \\
\hline \multicolumn{9}{|l|}{ Maternal behaviour } \\
\hline Activity & 97 & $20.15(1.73)$ & 191 & $20.14(1.31)$ & 585 & $22.50(0.80)$ & 0.916 & 0.280 \\
\hline Stimulation & 97 & $5.09(0.66)$ & 191 & $5.44(0.50)$ & 585 & $4.84(0.31)$ & 0.089 & 0.616 \\
\hline Engagement & 95 & $5.83(0.13)$ & 187 & $5.81(0.10)$ & 571 & $6.07(0.06)$ & 0.387 & 0.069 \\
\hline Flexibility & 97 & $6.32(0.13)$ & 191 & $6.46(0.10)$ & 585 & $6.58(0.06)$ & 0.159 & 0.205 \\
\hline Session & 97 & $641.22(23.54)$ & 191 & $656.01(17.80)$ & 585 & $684.66(10.98)$ & 0.026 & 0.216 \\
\hline
\end{tabular}

\section{Heavy cocaine exposure}

Table 7 gives the results for the three exposure groups (heavy, some, and none) for analysis 3 (unadjusted) and analysis 4 (adjusted for covariates). The adjusted group means from analysis 4 are presented. In the unadjusted analysis 3, heavy cocaine exposure was associated with lower infant arousal $(p=0.040)$ and shorter feeding sessions $(p=0.026)$ than some or no exposure. When covariates were included (analysis 4), these effects were no longer significant.

\section{Weight gain}

Given cocaine and opiate exposure effects on maternal and infant feeding behaviour, weight gain per week ( $g$ ) from birth to the 1 month feeding session was calculated and tested using analysis 2, which adjusted for covariates. This analysis was conducted separately for preterm $(<38$ weeks gestational age) and term infants, as preterm infants have different growth trajectories from term infants in part because of greater chronological age at the feeding session. For term infants, there were no significant differences in weight gain between cocaine exposed and non-exposed infants (230 v 222 $\mathrm{g} /$ week; $\mathrm{p}=0.532$ ). For preterm infants, however, cocaine exposed infants gained $25 \mathrm{~g} /$ week less than unexposed infants (202 v $227 \mathrm{~g} /$ week; p = 0.047). There were no significant differences in weight gain between opiate exposed and unexposed infants in term (226 $v 225 \mathrm{~g} /$ week; $\mathrm{p}=0.941)$ or preterm infants $(220 \vee 210 \mathrm{~g} /$ week; $\mathrm{p}=0.409)$. Taken together with the cocaine effects described above, these findings may suggest that cocaine-using mothers with preterm infants are the least flexible and engaging and have the shortest feeding sessions. No support for this hypothesis was found. The results of analysis of variance on these measures comparing term and preterm cocaine exposed infants showed no significant differences $(p>0.249$ in all cases $)$.

\section{Covariate effects}

We also examined whether covariates themselves were useful predictors of feeding behaviour. In both analyses 2 (cocaine and opiate effects) and 4 (level of cocaine exposure effects), mothers who were heavy tobacco users showed less flexibility $(p=0.023)$ and less engagement $(p=0.007)$ than sometime and non-users. Binge drinking was associated with a shorter feeding session $(p=0.023)$. Low birth weight was associated with more maternal stimulation $(p=0.001)$, less infant feeding competence $(p=0.050)$, more feeding problems $(p=0.010)$, and lower proportion of sucking $(p=0.010)$. Higher socioeconomic status was related to more maternal stimulation $(p=0.001)$ and engagement $(p=0.001)$, and more infant crying $(p=0.017)$. Conceptional age was related to longer sucking bursts $(p=0.013)$, higher proportion of sucking $(p=0.006)$, and higher arousal $(p=0.001)$. Maternal age was related to lower proportion of sucking $(p=0.016)$, more maternal stimulation $(p=0.001)$, and engagement $(p=0.001)$. Black mothers showed less stimulation $(\mathrm{p}=0.002)$, and their infants had a higher proportion of sucking $(p=0.026)$ and fewer sucking bursts $(p=0.010)$. Note that all main effects and covariate effects were partialled in these results.

\section{Birth weight as a mediator}

Analyses with covariates (analysis 2 and 4) were repeated excluding birth weight from the list of covariates because it has been argued that if cocaine affects birth weight, the inclusion of birth weight as a covariate will mask the effects of cocaine..$^{28}$ After birth weight had been excluded as a covariate, no additional (or fewer) cocaine effects were found relative to those previously reported with birth weight in the model.

\section{DISCUSSION}

Infants are born with a set of reflexes and endogenous sucking rhythms, including alternation of sucking bursts and pauses that guide and maintain feeding. ${ }^{26}{ }^{38}$ There are developmental changes in sucking rhythm within the first 2 weeks, with increased sucking and decreased pausing over time, suggesting greater feeding efficiency as infants mature. ${ }^{26}{ }^{36}$ The typical pattern for mothers over this period is to reduce feeding related activity-for example, stimulation with the bottle-as well as social stimulation-for example, vocalisations - that might interrupt the sucking rhythm, suggesting maternal sensitivity to increased infant maturity. 22636

\section{Cocaine exposure}

Previous clinical reports have described poor and disorganised feeding in neonates exposed to cocaine. ${ }^{19^{11}}$ Research using objective measurement of nutritive and non-nutritive sucking has reported decreased sucking (non-nutritive) among cocaine exposed neonates $(n=16)$ compared with nonexposed neonates, ${ }^{10}$ but these results were not replicated in a second study with a larger sample of neonates $(\mathrm{n}=71) \cdot{ }^{14} \mathrm{In}$ our study, cocaine exposure was not associated with poor sucking or feeding, and cocaine exposed infants did not show disorganised, non-optimal sucking rhythms (sucking bursts, interburst interval, bursts per minute) or increased feeding problems. However, we cannot rule out atypical sucking patterns, such as reduced sucking pressure, that could only be 
detected by objective measurement systems and computer analysis or could have occurred during the early neonatal period and resolved by the 1 month feeding session.

Poor feeding could also result from the type and timing of stimulation provided by mothers who might be less responsive to their infants' cues or feeding difficulties. ${ }^{136}{ }^{39-41}$ Previous studies found that cocaine exposed infants gave fewer cues and were less responsive to their mothers, and their mothers were less sensitive to their infants' cues as newborns, ${ }^{5}$ and as a consequence there were more dyadic conflicts as early as 2 months and up to 24 months of age. ${ }^{6}{ }^{12}$ However, findings are not replicated in a sample of infants $7-16$ weeks of age, ${ }^{15}$ and in this large, rigorously studied sample, cocaine exposed infants were not less competent or less responsive feeders than non-exposed infants. Nevertheless, their mothers were less flexible in response to their infants' cues, were less warm or engaged with their infants, and ended the feeding session sooner. Further, cocaine-using mothers who also used opiates were more likely to be disengaged from their infants as well as less stimulating during feeding. These maternal findings suggest less sensitivity and involvement with the infant during the session. Because the infants did not compensate for the shorter feed with a higher proportion of sucking or longer sucking bursts, shorter feeding sessions resulted in less overall feeding opportunity.

Although we acknowledge that there are multiple determinants of weight gain, we found that cocaine exposed preterm infants gained less from birth to 44 weeks postmenstrual age than comparison preterm infants after adjustment for birth weight, conceptional age (gestational + chronological age), as well as polydrug exposure, race, maternal age, and socioeconomic status. As the feeding style of cocaine-using mothers was similar for preterm and term infants, these findings suggest that preterm, cocaine exposed infants may be more vulnerable to maternal behaviour that limits feeding opportunity than term infants.

\section{Opiate exposure}

Previous research has documented less sucking, reduced sucking pressure, and lower nutrient consumption in opiate exposed neonates. ${ }^{78}$ In our study, opiate exposed infants did not suck less during the feeding session. Rather, they showed prolonged sucking with fewer pauses, more feeding problems such as spitting up and refusal, and increased arousal. Opiate exposed infants who were also exposed to cocaine were most likely to be aroused. These behaviours are consistent with clinical reports of opiate withdrawal. ${ }^{7}$ We cannot determine in this study whether opiate exposed infants had poorer nutrient consumption than non-exposed infants. However, both term and preterm opiate exposed infants showed no decrements in weight gain. Although there were increased arousal and feeding problems, opiate exposed infants also had longer sucking bursts and fewer breaks, which are consistent with greater energy intake.

Opiate-using mothers showed more feeding activity, but these behaviours did not account for their infants' heightened sucking, although they may have contributed to the increased arousal and feeding problems shown by the infants. Opiateusing mothers also appeared to be less flexible and more stimulating than non-using mothers, but these effects were reduced after adjustment. However, opiate-using mothers who did not use cocaine were more likely to stimulate their infants than non-users after adjustment. By 1 month, increased maternal activity not linked to infant feeding problems suggests less sensitivity to infant cues ${ }^{39}$ by opiate-using mothers.

There were no findings in common for cocaine and opiate involved infants and mothers. The lack of common effects suggests, as might be expected, that cocaine and opiates have specific implications for the dyad. However, the combination of cocaine and opiates appeared to exacerbate some of the individual drug effects. Infants exposed to both drugs were the most aroused. Mothers who used both drugs were the least flexible and engaging with the infants.

\section{Heavy cocaine exposure}

On unadjusted analyses, heavy cocaine exposure was not associated with any feeding behaviour except low arousal and the length of the feeding session; however, neither effect was significant after adjustment for covariates. These results provide no evidence that cocaine exposure or even heavy cocaine exposure compromises infant sucking and feeding behaviour.

\section{Substance exposure in context}

It is important to note that maternal and infant feeding behaviour is determined by many factors. Consistent with previous research, ${ }^{23}$ low birth weight in this study was associated with poorer feeding behaviour including lower proportion of sucking, less feeding competence, and more feeding problems at 1 month of adjusted age, suggesting less maturity even after age correction. As expected, ${ }^{36}$ conceptional age, which combines gestational age and chronological age, predicted more mature feeding patterns. There was no association between low birth weight and length of session or maternal measures of feeding activity and flexibility. However, mothers of preterm infants showed increased stimulation, which may reflect greater maternal perception of infant vulnerability. ${ }^{236}$ Birth weight is probably not a mediator variable ${ }^{42}$, meaning that cocaine effects could be masked by the inclusion of birth weight. Firstly, significant cocaine and birth weight effects did not overlap on any feeding measure. Secondly, the results of analyses with and without birth weight in the model generated the same cocaine findings. Taken together, these observations suggest that birth weight and cocaine effects were independent contributors to feeding behaviour.

Higher socioeconomic status was also associated with more maternal verbal and physical contact as well as emotional engagement. ${ }^{133}$ Engagement evaluates the quality of attention and affection, suggesting that the stimulation provided by these higher socioeconomic status mothers was also warm and responsive. Older mothers were also more stimulating and engaging, ${ }^{13}$ but their babies spent less time sucking over the session, suggesting that the increased interaction in this case could have reduced feeding efficiency, unlike socioeconomic status. Black mothers, who stimulated their infants less than white or Hispanic mothers, had infants who spent more time sucking over the session with fewer breaks, suggesting a pattern of maximum feeding efficiency with little interaction.

Previous research on neonates exposed to alcohol/nicotine found poor sucking, lower sucking pressure, and less sucking. ${ }^{43-45}$ In our study, exposure to tobacco, alcohol, or marijuana was not associated with any compromise of infant sucking or feeding behaviour. However, heavy tobacco use was related to decreased maternal flexibility and engagement, and binge drinking was associated with a decreased length of session, independent of cocaine effects. There were no marijuana effects in the study.

The interpretation of this study is limited by efforts to maintain a natural feeding session without intrusion by equipment or staff. Without specialised equipment, we could not detect high amplitude sucking, sucking pressure, and sucking speed, which have been found to be sensitive to drug exposure and related to energy intake and future weight gain. ${ }^{343}$ Our rationale was that the burst and pause pattern of infant sucking that is observable to coders is particularly salient to mothers and may be more closely linked to her levels of activity and stimulation. ${ }^{26}$ Further, we did not obtain the exact amount of formula the infant drank during the feeding to 
determine feeding efficiency, but note that previous studies reported a significant association between energy intake and infant behaviour of the type measured in this study. ${ }^{16}$ However, our conclusions on feeding efficiency would have benefited from direct measurement of energy intake during the feeding. We excluded breast feeding dyads because mothers may be uncomfortable being videotaped while feeding, some of our feeding measures would not be appropriate, and the number of mothers who breast fed was small. Thus, we cannot assume that our findings apply to drug-using mothers who breast feed their infants. Further, although the sample was recruited randomly at four separate hospitals, the characteristics of the hospital and self selected participation in the follow up may also limit generalisability to other ethnic and/or income groups. Given that we hypothesised negative effects of drug exposure on infant and maternal behaviour a priori and expected that these effects would be subtle, we did not correct for the possibility of a type I error on primary planned analyses. However, multiple comparison corrections were used for all post hoc tests. The systematic pattern of findings across analyses suggests that the results are not due to chance. Given the large sample size, those effects close to the significance cut off of $p<0.05$ are of small effect size, which is expected in analysis of prenatal drug exposure. To correct for multiple tests would invite type II error, in which true effects are missed ${ }^{46}$ Findings of subtle effects not only enhance scientific understanding but can have enormous impact on public policy and treatment. ${ }^{47}$

\section{Conclusion}

Prenatal exposure to cocaine and opiates had unique implications for feeding behaviour in 1 month old infants and their mothers, after polydrug use, birth weight, conceptional age, and social factors were controlled for. Contrary to previous concerns, ${ }^{15}{ }^{9-12}$ cocaine exposed infants did not show feeding difficulties in this study, whereas opiate exposed infants showed prolonged sucking and other problems consistent with signs of opiate withdrawal. Cocaine-using mothers were less engaged and less flexible in response to their infants' cues, ${ }^{5}$ whereas opiate-using mothers showed higher levels of feeding activity, independent of the feeding problems shown by their infants. Finally, the impact of using both cocaine and opiates and the additive effect of tobacco suggests that infants whose mothers use multiple drugs are likely to be the most at risk of insensitive, inflexible parenting during feeding. Maternal behaviour that overstimulates, controls, and potentially limits feeding opportunity may compromise energy intake and future weight gain and lead to mother-infant conflict. ${ }^{12}$ Concerns about feeding behaviour in cocaine exposed infants may reflect problems related to the feeding interaction rather than to feeding problems in the infant. Early feeding interactions may reflect problems in the developing mother-infant relationship that could have long term implications for poor weight gain and subsequent relationship difficulties, particularly for preterm infants.

\section{ACKNOWLEDGEMENTS}

This work was supported by the National Institute on Child Health and Human Development (NICHD contract number NOl-HD-2-3159) and through cooperative agreements (U10 HD 27904 to BML; U10 HD 21397 to CRB; U10 HD 21385 to SS; U10 HD 27856 to HB) and Intraagency agreements with the National Institute on Drug Abuse (NIDA), Administration on Children, Youth and Families (ACYF), and the Center for Substance Abuse Treatment (CSAT).

\section{Authors' affiliations}

L L LaGasse, B M Lester, J Liu, Brown Medical School, Women \& Infant's Hospital and Bradley Hospital, Providence, RI, USA

D Messinger, University of Miami, Miami, FL, USA

R Seifer, Brown Medical School and Bradley Hospital, Providence
E Z Tronick, Harvard University Medical School and Children's Hospital, Boston, MA, USA

C R Baver, University of Miami School of Medicine, Miami

S Shankaran, Wayne State University School of Medicine, Detroit, MI, USA

H S Bada, University of Kentucky College of Medicine, Lexington, KY, USA

L L Wright, National Institute of Child Health and Human Development (NICHD), Bethesda, MD, USA

V L Smeriglio, National Institute on Drug Abuse (NIDA), Bethesda

L P Finnegan, Office of Research on Women's Health, National Institutes of Health, Bethesda

P L Maza, Administration on Children, Youth and Families (ACYF), Washington, DC, USA

\section{REFERENCES}

1 Sanders-Phillips K. Infant feeding behavior and caretaker-infant relationships in Black families. J Compar Family Studies 1998;29:161-71.

2 Weiss SJ, Wilson P, Hertenstein M, et al. The tactile context of a mother's caregiving: implications for attachment of low birth weight infants. Infant Behav Dev 2000;23:91-1 11

3 Agras WS, Kraemer HC, Berkowitz Rl, et al. Does a vigorous feeding style influence early development of adiposity? J Pediatr 1987;1 10:799-804.

4 Dubignon J, Cooper D. Good and poor feeding behavior in the neonatal period. Infant Behav Dev 1980;3:395-408

5 Barabach LM, Glazer G, Norris SC. Maternal perception and parent-infant interaction of vulnerable cocaine-exposed couplets. J Perinat Neonatal Nurs 1992;5:76-84

6 Das Eiden RD. Maternal substance use and mother-infant feeding interactions. Infant Mental Health J 2001;22:497-511.

7 Kron RE, Litt M, Finnegan LP. Narcotic addiction in the newborn: differences in behavior generated by methadone and heroin. Int J Clin Pharmacol 1975;12:63-9.

8 Kron RE, Litt M, Phoenix MD, et al. Neonatal narcotic abstinence: effects of pharmacotherapeutic agents and maternal drug usage on nutritive sucking behavior. Pediatrics 1976;88:637-41.

9 Madden JD, Payne RF, Miller S. Maternal cocaine abuse and effect on the newborn. Pediatrics 1986;77:209-11.

10 Maone RR, Mattes RD, Beauchamp GK. Cocaine-exposed newborns show an exaggerated sucking response to sucrose. Physiol Behav 1992;51:487-91.

11 Oro AS, Dixon SD. Perinatal cocaine and methamphetamine exposure: maternal and neonatal correlates. J Pediatr 1987;111:571-8.

12 Platzman KA, Coles CC, Lynch ME, et al. Assessment of the caregiving environment and infant functioning in polydrug families: use of a structured clinical interview. Infant Mental Health J 2001;22:351-73.

13 Hadeed AJ, Siegel SR. Maternal cocaine use during pregnancy: effect on the newborn infant. Pediatrics 1989;84:205-10.

14 Martin JC, Barr HM, Martin DC, et al. Neonatal neurobehavioral outcome following prenatal exposure to cocaine. Neurotoxicol Terato 1996; 18:617-25.

15 Neuspiel DR, Hamel SC, Hochberg E, et al. Maternal cocaine use and infant behavior. Neurotoxicol Teratol 1991;24:229-33.

16 Mullen MK, Garcia Coll C, Muriel AC, et al. Mother-infant feeding interaction in full-term small-for-gestational-age infants. J Pediatr 1988;112:143-8.

17 Baver CR, Shankaran S, Bada H, et al. The Maternal Lifestyle Study: drug exposure during pregnancy and short-term maternal outcomes. Am J Obstet Gynecol 2002;186:487-95.

18 EISohly MA, Stanford DF, Murphy TP, et al. Immunoassay and GC-MS procedures for the analysis of drugs of abuse in meconium. J Analyt Toxicol 1999;23:436-45.

19 Lester BM, ElSohly MA, Wright LL, et al. The Maternal Lifestyle Study: drug use by meconium toxicology and maternal self-report. Pediatrics 2001; 107:309-17.

20 Lester BM, Tronick EZ, Mayes L, et al. Neurodevelopmental consortium, the NICHD Neonatal research network. A neurodevelopmental follow-up battery for substance exposed infants. Pediatr Res 1994;35:23A.

21 Garcia Coll CT, Halpern L, Seifer R, et al. Behavioral intervention and post-natal growth in full-term intrauterine growth retarded (IUGR) infants. Early Human Dev 1996:46:105-16.

22 Meyer EC, Garcia Coll CT, Lester BM, et al. Family-based intervention improves maternal psychological well-being and feeding interaction of preterm infants. Pediatrics 1994;93:241-6.

23 Shankaran S, Katsikiotis V, Baver CR, et al. Patterns of substance use in term pregnancy and effect on birthweight (BW) and head circumference (HC) [abstract]. Pediatr Res 1997;41:177A.

24 LaGasse LL, Seifer R, Wright LL, et al. The Maternal Lifestyle Study (MLS): the caretaking environment of infants exposed to cocaine/opiates. Pediatr Res 1999:45:247A.

25 McLellan AT, Luborsky L, Cacciola J, et al. An improved diagnostic evaluation instrument for substance abuse patients: The Addiction Severity Index. J Nervous Mental Dis 1980;168:26-33.

26 Kaye K, Wells A. Mothers' jiggling and the burst-pause pattern in neonatal feeding. Infant Behav Dev 1980;3:29-46.

27 Brazelton TB. Neonatal behavioral assessment scale. 2nd ed. London: Spastics International Medical Publications, 1984:17. 
28 Jacobson SW, Jacobson JL, Sokol RJ, et al. New evidence for neurobehavioral effects of in utero cocaine exposure. J Pediatr 1996;1 29:581-90

29 Jacobsen JL, Jacobson SW. Methodological issues in human behavioral teratology. In: Rovee-Collier C, Lipsitt L, eds. Advances in infancy research. Norwood, NJ: ABLEX, 1990:111-48.

30 LaGasse LL, Seifer R, Lester BM. Interpreting research on prenatal substance exposure in the context of multiple confounding factors. Clin Perinatol 1999;26:39-54

31 Leon DA. Failed or misleading adjustment for confounding. Lancet 1993:342:479-81.

32 Richardson GA, Day NL. Studies of prenatal cocaine exposure: assessing the influence of extraneous variables. J Drug Issues 1999;29:225-36.

33 Walker LO, Crain H, Thompson E. Mothering behavior and maternal role attainment during the postpartum period. Nurs Res 1986;35:352-5.

34 Field T, Morrow C, Adlestein D. Depressed mothers' perceptions of infant behavior. Infant Behav Dev 1993;16:99-108.

35 Lanzi RG, Pascoe JM, Keltner B, et al. Correlates of maternal depressive symptoms in a National Head Start Program sample. Arch Pediatr Adolesc Med 1999;153:801-4.

36 DiVitto B, Goldberg S. Talking and sucking: infant feeding behavior and parent stimulation in dyads with different medical histories. Infant Behav Dev 1983;6:157-65.

37 Jacobson JL, Jacobson SW, Sokol RJ. Effects of prenatal exposure to alcohol, smoking and illicit drugs on postpartum somatic growth. Alcohol: Clin Exp Res 1994;18:317-23.
38 Hafstrom $M$, Kiellmer I. Non-nutritive sucking in the healthy pre-term infant. Early Hum Dev 2000;60:13-24.

39 Field T. Maternal stimulation during infant feeding. Dev Psychol 1977;13:539-40

40 Frank DA, Bresnahan K, Zuckerman BS. Maternal cocaine use: impact on child health and development. In: Barness LA, ed. Advances in pediatrics. St Louis, Mo: Mosby, 1993;40

41 Klaus MH, Kennell JH, Plumb N, et al. Human maternal behavior at first contact with her young. Pediatrics 1970;46:187-92.

42 Baron RM, Kenny DA. The moderator-mediator variable distinction in social psychological research: conceptual, strategic and statistical considerations. J Pers Soc Psychol 1986;51:1173.

43 Martin DC, Martin JC, Streissguth AP, et al. Sucking frequency and amplitude in newborns as a function of maternal drinking and smoking. In: Galanter M, ed. Currents in Alcoholism, Biomedical Issues and Clinical Effects of Alcoholism. New York: Grune \& Stratton, 1979;5:351-66.

44 Ouellette EM, Rosett HL, Rosman NP, et al. Adverse effects on offspring of maternal alcohol abuse during pregnancy. N Engl J Med 1977:297:528-30.

45 Streissguth AP, Barr HM, Martin DC, et al. Effects of maternal alcohol, nicotine, and caffeine use during pregnancy on infant mental and motor development at eight months. Alcohol: Clin Exp Res 1980;4:152-63.

46 Rothman KJ. No adjustments are needed for multiple comparisons. Epidemiology 1990;1:43-6.

47 Lester BM, LaGasse LL, Seifer R. Cocaine exposure and children: the meaning of subtle effects. Science 1998;282:633-4.

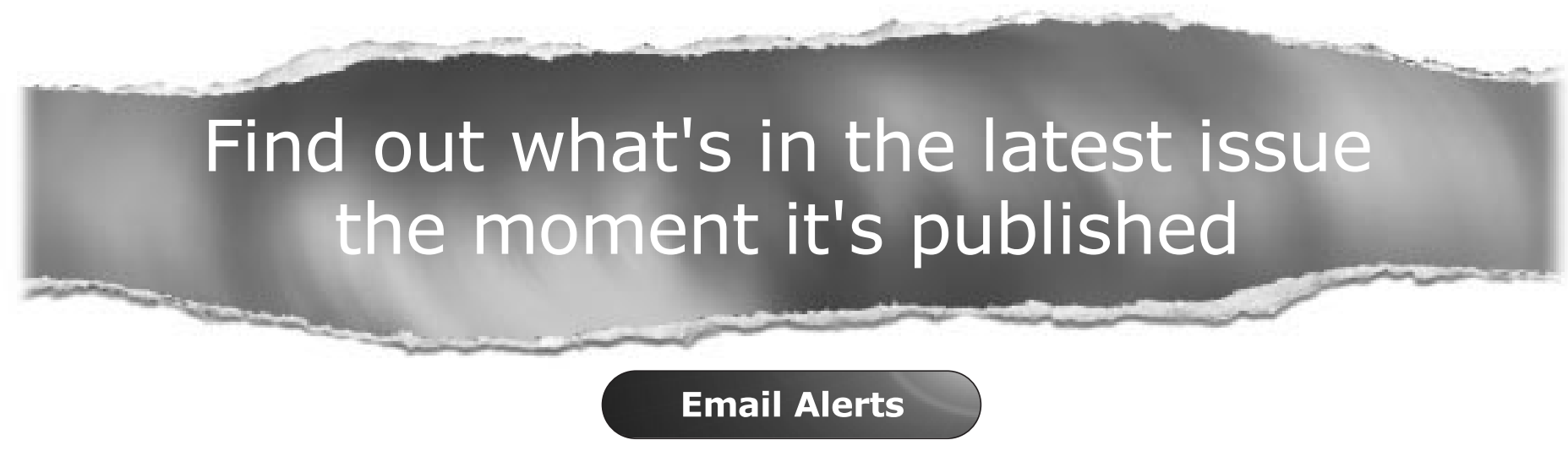

Sign up to receive the table of contents by email every month. You can select from three alerts:

Table of Contents (full), TOC Awareness (notice only); Archives of Disease in Childhood related announcements.

www.archdischild.com 